\title{
A dielectric/metal layer system as chemical sensor
}

\author{
Helene E. de Bruijn, Aufried T.M. Lenferink, Rob P.H. Kooyman and Jan Greve \\ University of Twente, Faculty of Applied Physics, P.O. Box 217, 7500 AE Enschede, Netherlands
}

Received 11 June 1991

\begin{abstract}
Simulations and experiments are presented of a dielectric/metal layer structure as a chemical sensor, that exhibits a sensitivity comparable to that found for SPR sensors, but without their drawbacks. The sensitivity to both s- and p-type light permits the measurements of additional parameters of the chemical system under study.
\end{abstract}

\section{Introduction}

In recent years an increasing interest has developed in the application of the Surface Plasmon Resonance (SPR) phenomenon to the design of optochemical sensors and to devices where a microscopic image of a surface phase structure can be obtained [1]. An important reason for the use of SPR in these sensors is the large field amplification at the metaldielectric interface resulting in a high sensitivity to changing refractive index profiles near the interface.

However, from a practical point of view the presence of a thin metal layer poses several problems, such as its stability to chemical attack and lack of adhesion to the underlying substrate. Another difficulty can be that the metal layer precludes an efficient immobilization of receptor molecules required for a selective binding of the analyte.

In this note we will indicate that in a slightly different optical configuration these problems can be easily overcome, while the intrinsic sensitivity of the SPR device is largely retained.

\section{Theory}

Consider a structure as depicted in fig. 1 , where a thin metal layer 2 is sandwiched between a prism 1 and a dielectric layer 3 with thickness $O(\lambda)$. Otto et al. [2] have shown that in this structure several resonance minima in the reflectance can be obtained and that one of these minima corresponds to the ex-

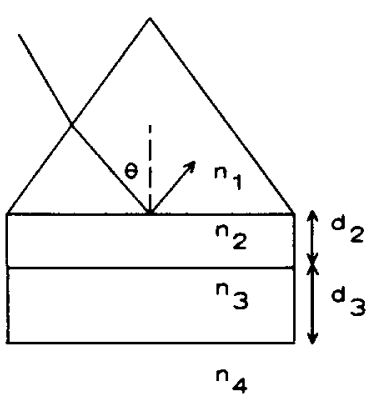

Fig. 1. The Kretschmann configuration with the prism $\left(n_{1}\right)$, metal layer $\left(n_{2}, d_{2}\right)$ and optional dielectric layer $\left(n_{3}, d_{3}\right)$ in an environment $\left(n_{4}\right)$.

citation of the surface plasmon. The optical response of this configuration can be analyzed using Fresnel's equations [3]. A typical result of such calculations is shown in fig. 2. As these calculations were done for one angle, corresponding to the plasmon angle at $d_{3}=0$, it is not surprising that we find a periodicity $d_{3}^{*}$ in the behavior of the reflectance as a function of $d_{3}$, corresponding to an additional optical pathlength $\lambda=2 n_{3} d_{3}^{*} \cos \theta_{3}$. As in general

$$
\begin{aligned}
r_{234} & =\frac{r_{23}+r_{34} \exp \left(2 \mathrm{i} k_{z 3} d_{3}\right)}{1+r_{23} r_{34} \exp \left(2 \mathrm{i} k_{z 3} d_{3}\right)} \\
& =\frac{r_{23}+r_{34} \exp \left[2 \mathrm{i} k_{z 3}\left(d_{3}^{0}+m d_{3}^{*}\right)\right]}{1+r_{23} r_{34} \exp \left[2 \mathrm{i} k_{z 3}\left(d_{3}^{0}+m d_{3}^{*}\right)\right]},
\end{aligned}
$$

a thickness change of layer 3 with $m d_{3}^{*}$ only causes a phase change $2 \pi m$, which does not influence the reflection coefficient. 


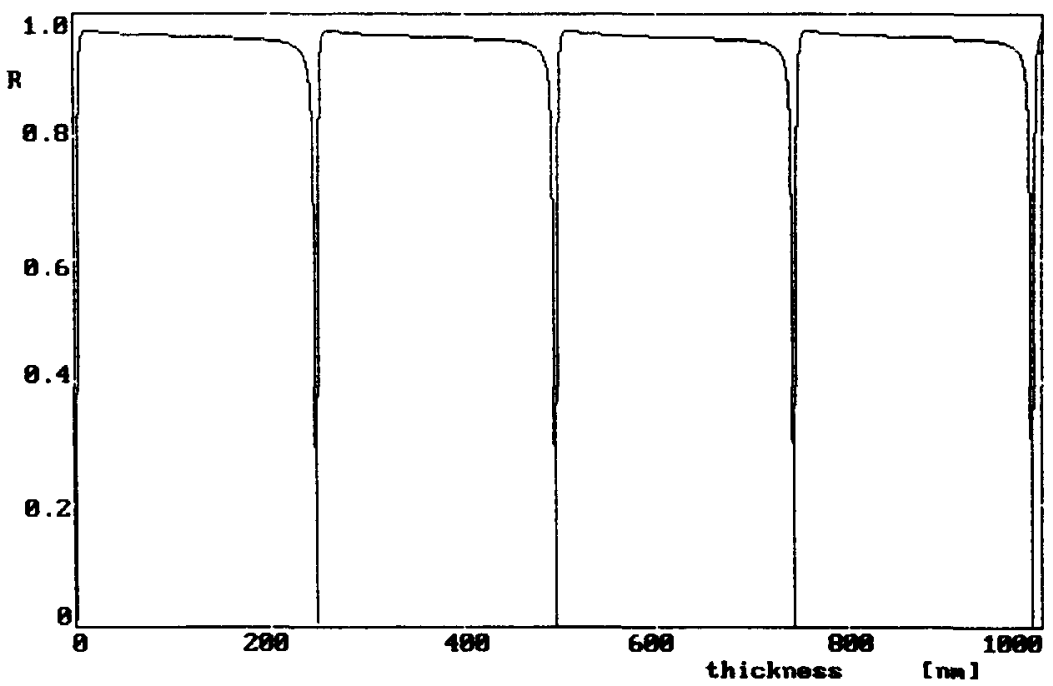

Fig. 2. Calculated reflection coefficient $R$ of $53 \mathrm{~nm}$ silver at $\theta=42.96^{\circ}$ as a function of thickness $d_{3}$ of a dielectric layer $n_{3}=1.63$. The period is $248 \mathrm{~nm}(\lambda=632.8 \mathrm{~nm})$.

Fig. 3a gives the resonance angle as a function of the thickness of layer 3 . The angle $\theta=42.96^{\circ}$ corresponds to the situation of fig. 2 . A thin dielectric layer just causes a shift in resonance angle as is known from sensor applications. Each resonance angle has a period $d_{3}^{*}$ at which resonance occurs, which increases for increasing angle. The increment leads to overlap of the different order numbers $m$ for a thick layer 3. This means that for the thicker layers more than one resonance angle is found.

With s-polarization one can obtain resonance curves at different angles [2], which are given in fig. $3 b$. It shows that a minimum thickness of layer 3 is necessary to get resonance. For p-polarization the minimum reflection remains low for all resonance angles whereas for s-polarization the minimum reflection increases with increasing resonance angle. This is why Otto [2] could not measure the lower order TE resonances.

The calculated field distributions in fig. 4 show that the field distribution in the metal and bulk material at the plasmon angle of bare silver is similar to that of the silver with a dielectric layer. In a direction perpendicular to the interfaces the field in the dielectric layer is a standing wave.

The calculations apply to plane electromagnetic waves. In the experiments a laser beam is used and the approximation to plane waves is only valid if the lateral displacement in layer in layer 3: $2 d_{3} \sin \theta_{3}$ is small compared to the beam width.

\section{Sensitivity considerations}

When the p-polarized reflectance of a bare metal layer is determined as a function of the angle of incidence one gets a resonance curve with a certain halfwidth $\Delta \theta$. The plasmon-angle $\theta_{\mathrm{p}}$ corresponds to certain thicknesses $d_{3}$ of layer 3 for which the same minimum at this angle can be found. Simulations show a decreasing halfwidth for increasing thickness $d_{3}$, this smaller halfwidth might lead to a better sensitivity when SPR is used for sensors. For the sensitivity the shift in plasmon angle due to an additional dielectric layer is also important [4].

Table 1 gives the resonance width of the reflection curves of a bare silver layer and silver layers with $d_{3}^{*}$ and $2 d_{3}^{*}$ thick dielectric layers; furthermore the shift in resonance angle due to an additional $2 \mathrm{~nm}$ layer ( $n=1.41)$ is calculated. The bare silver layer has a broader resonance curve, but the angle shift due to the added layer is larger. This means that the sensitivity decreases with increasing order number $m$, when the sensor measures the angle shift directly. Some sensors measure the reflection change at a fixed angle of incidence and for those sensors the sensi- 

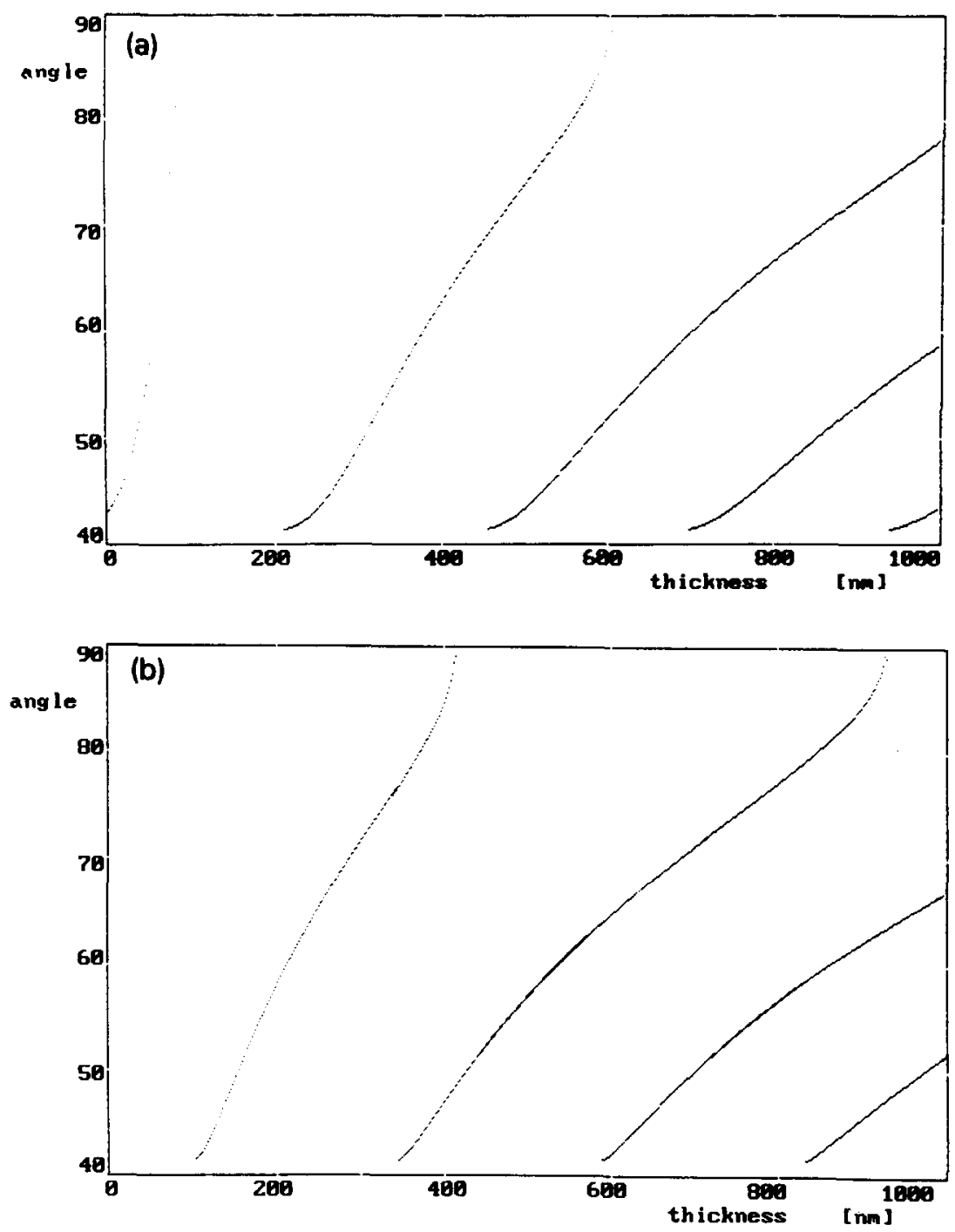

Fig. 3. The calculated resonance angle of $53 \mathrm{~nm}$ silver $(\epsilon=-16+0.6 \mathrm{i})$ as a function of the thickness $d_{3}$ of a dielectric layer $n_{3}=1.63$ on top of the silver for p-polarization (a) and s-polarization (b).

tivity does not change much as the smaller angle shift is compensated by the steeper reflection curve.

For s-polarization a layer of $116 \mathrm{~nm} n_{3}=1.63$ on top of the silver layer leads to the same resonance angle as bare silver for p-polarization. The shift due to a $2 \mathrm{~nm}$ layer $n=1.41$ is $0.18^{\circ}$ and the halfwidth is $0.29^{\circ}$. These figures are very close to those of bare silver at p-polarization and the sensor will have the same sensitivity. Higher order layer thicknesses will lead to similar situations as for p-polarization.

\section{Experiments}

The above results about a dielectric layer on top of the metal were verified experimentally by spinning photoresist layers $\left(n_{3}=1.63\right)$ on top a silver layer. 

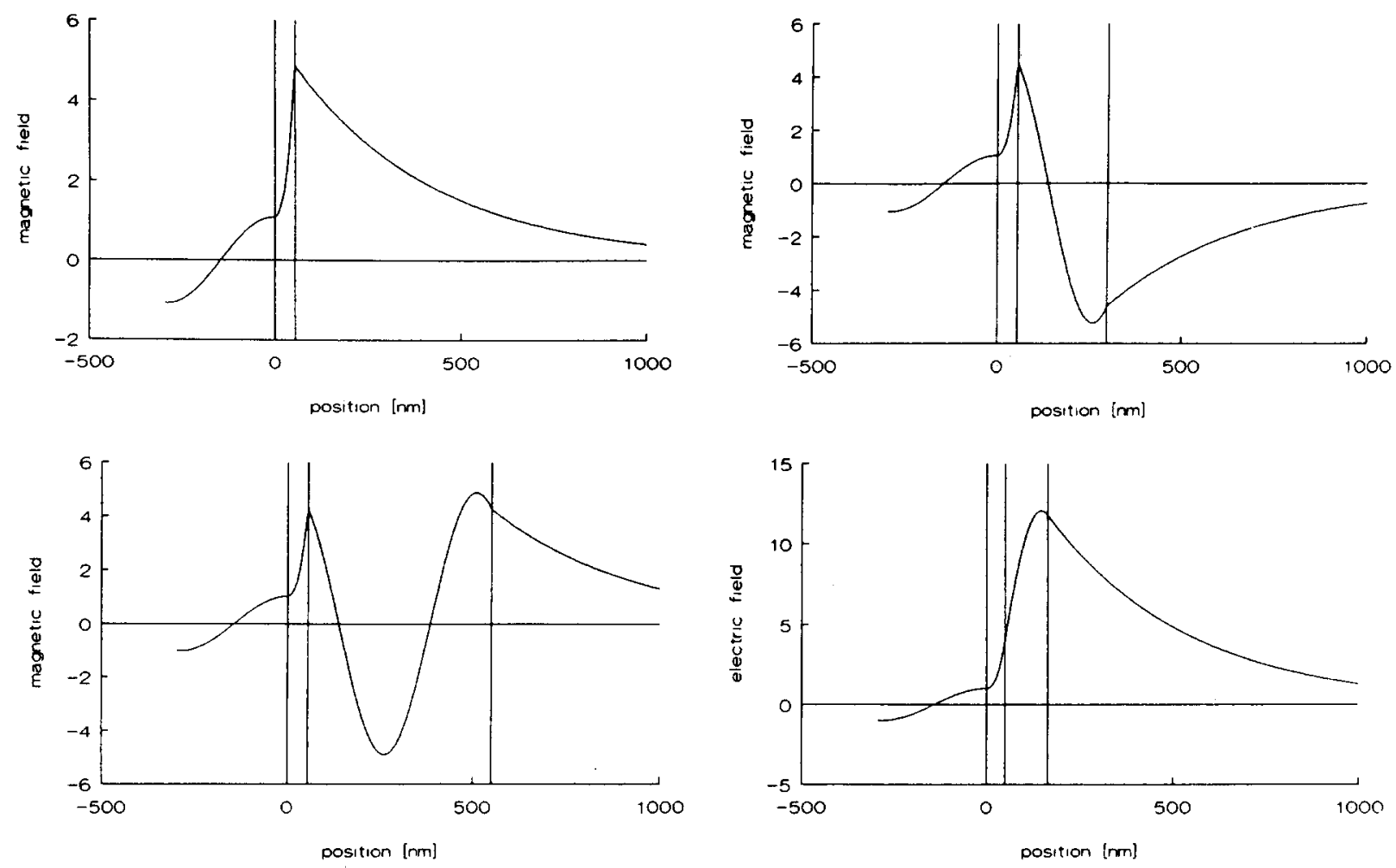

Fig. 4. The calculated field distribution in the layer system. Position 0 corresponds with the prism silver interface. The calculations are done for $53 \mathrm{~nm}$ silver without a dielectric (a), with $248 \mathrm{~nm} n_{3}=1.63$ (b), with $496 \mathrm{~nm} n_{3}=1.63$ (c) all for p-polarization and for 47.5 $\mathrm{nm}$ silver with $116 \mathrm{~nm} n_{3}=1.63$ for s-polarization (d).

Table 1

Resonance width $\Delta \theta$ of reflection curves and shift in resonance angle due to a $2 \mathrm{~nm}$ layer $n=1.41$ on a silver layer with the specified thickness of photoresist.

\begin{tabular}{lcll}
\hline Polarization & $\begin{array}{l}\text { Thickness } \\
\text { photoresist } \\
{[\mathrm{nm}]}\end{array}$ & $\Delta \theta$ & $\begin{array}{l}\text { Angle } \\
\text { shift }\end{array}$ \\
\hline p & 0 & $0.30^{\circ}$ & $0.17^{\circ}$ \\
p & 248 & $0.22^{\circ}$ & $0.12^{\circ}$ \\
p & 496 & $0.18^{\circ}$ & $0.10^{\circ}$ \\
s & 116 & $0.29^{\circ}$ & $0.18^{\circ}$ \\
\hline
\end{tabular}

Calculations predict that a thickness $d_{3}^{*}=248 \mathrm{~nm}$ exhibits a reflectance minimum at an angle corresponding to the plasmon angle of bare silver (see fig. 2 ). We tried to make layers of 248 and $496 \mathrm{~nm}$. The actual thickness was measured with a surface profiler (Dektak 3030) with an accuracy of $5 \mathrm{~nm}$.

The experimental reflection curves, obtained with an experimental setup described elsewhere [5], are presented in fig. 5. The resonance angle of the 500 $\mathrm{nm}$ layer is close to the angle of the bare silver, implying that indeed $d_{3}^{*}$ is about $250 \mathrm{~nm}$. We also found two resonance angles as predicted in fig. 3.

\section{Discussion}

We have shown that an optical configuration as shown in fig. 1 exhibits a sensitivity to refractive index changes near the $3 / 4$ interface which is comparable to that obtained by SPR devices.

The presence of the dielectric layer, the nature of which can be chosen within wide optical or chemical limits, makes it possible to adapt the interface to the requirements of the receptor molecule. Simultaneously, the metal layer is effectively protected against chemical attack. 


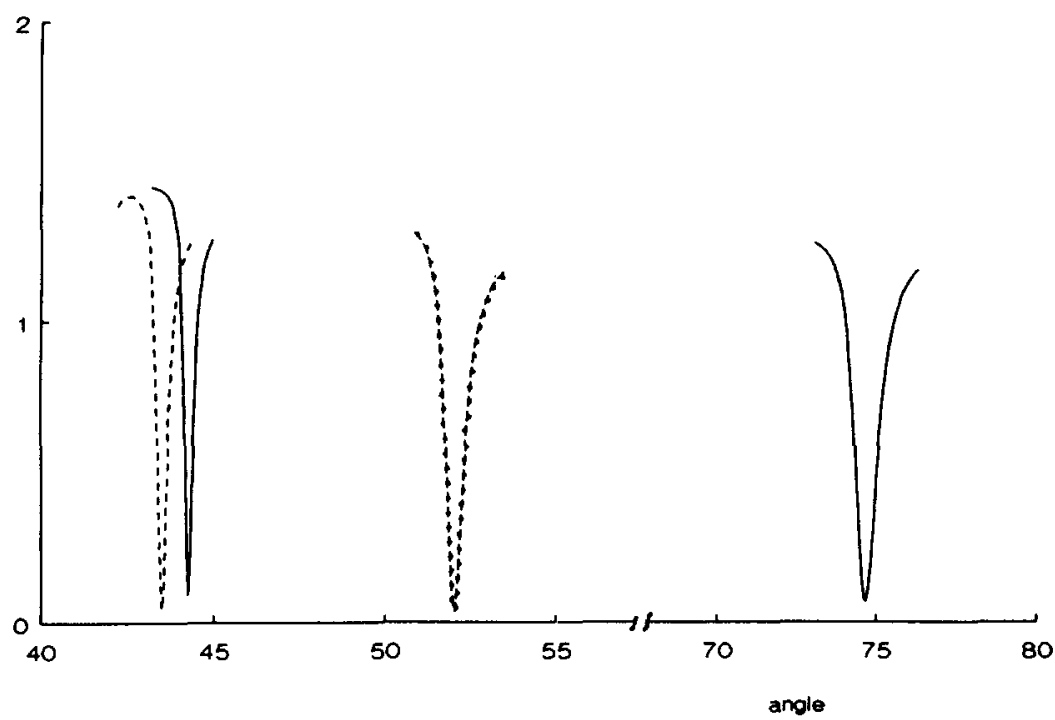

Fig. 5. The experimental reflection curves of bare silver $\left(--, \theta_{\mathrm{p}}=43.50^{\circ}\right)$, silver $+308 \mathrm{~nm}$ photoresist $\left(\cdots, \theta_{\mathrm{p}}=52.06^{\circ}\right)$ and silver +500 nm photoresist $\left(-, \theta_{\mathrm{p}}=44.24^{\circ}\right.$ and $74.66^{\circ}$ ).

Another possible application is integrating the receptor molecule in the dielectric layer 3 and leading the analyte through this layer. For gas sensors this may be done by diffusion; for fluid sensing a flow system with the dielectric layer as flow channel is necessary. In this way the high amplitude of the electromagnetic field in layer 3 is used.

The different changes of the resonance angle due to an additional layer 4 for p-polarized resonance and s-polarized resonance can be used to characterize layer 4 with its dielectric constant and thickness [6].

Also in microscopic devices to study phase objects the different $\mathrm{p}$ - and s-polarized resonances can provide additional information compared to the SPR microscope.

\section{References}

[1] K. Welford, Opt. Quan. El. 23 (1991) 1.

[2] A. Otto and W. Sohler, Optics Comm. 3 (1971) 254.

[3] J.R. Reitz, F.J. Milford and R.W. Christy, Foundations of electromagnetic theory, third edition (Addison Wesley, New York, 1979) p. 394.

[4] R.P.H. Kooyman, H. Kolkman, J. van Gent and J. Greve, Anal. Chim. Acta 213 ( 1988$) 35$.

[5] H.E. de Bruijn, B.S.F. Altenburg, R.P.H. Kooyman and J. Greve, Optics Comm. 82 (1991) 425.

[6] W. Lukosz, D. Clerc, Ph.M. Nellen and Ch. Stamm, Eurosensors IV, book of abstracts (Karlsruhe, 1990) B.7.2. 